\title{
Searching for ' ' and model discrimination in Atlas
}

\author{
Benjamin Trocme ${ }^{* \dagger}$ \\ LPSC, France \\ E-mail: benjamin.trocmedlpsc.in2p3.fr
}

If a heavy $Z^{\prime}$ boson exists, it could be one of the first particles to be discovered at the LHC, since its decay into two leptons will provide a clean signature. Interpreting the resonance may be more complicated. Here, we summarize a full detector simulation study on the possibility of distinguishing between models of $Z^{\prime}$ like narrow resonances, using as observables the width, cross section and forward-backward asymmetry.

International Europhysics Conference on High Energy Physics

July 21st - 27th 2005

Lisboa, Portugal

\footnotetext{
* Speaker.

$\dagger$ on behalf of the ATLAS collaboration
} 
The presence of additional neutral spin 1 bosons (denoted as $Z^{\prime}$ in this note) is predicted in many extensions of the Standard Model: models derived from the breakings of the $E_{6}$ gauge group, left-right symmetric models, extra dimensions models...The current most stringent limits are derived from the recent searches at the Tevatron[1][2] and range between $600 \mathrm{GeV}$ and $800 \mathrm{GeV}$, depending on the assumed model. The Large Hadron Collider at Cern whose first collisions are expected in fall 2007, should open a new frontier in this field : bosons of mass up to $4 \mathrm{TeV}$ could in fact be discovered in one year of data taking at the design luminosity (see [3] for instance).

If a $Z^{\prime}$ is discovered, the next challenge will consist in determining the underlying theory at the origin of this new gauge boson; this can be done by precisely measuring specific observables such as : natural decay width, rapidity, forward backward asymmetry... The measurement of the latter, not trivial at a hadronic collider, is detailed in this note, in the case of a $Z^{\prime}$ decay to electron-positron.

\section{Angular considerations}

Due to the spin 1 nature of the $Z^{\prime}$, its production cross section strongly depends on $\cos \theta^{\star}$, the angle between the final lepton and the initial quark in the rest frame, with the behaviour:

$$
\frac{d \sigma}{d \cos \theta^{\star}} \propto \frac{3}{8}\left(1+\cos ^{2} \theta^{\star}\right)+A_{F B} \cos \theta^{\star}
$$

Checking this dependence can be a way to probe the spin value of the new gauge boson but also to disentangle between spin 1 models by precisely measuring the $A_{F B}$ parameter. This measurement is challenging at the LHC, where the direction of the initial quark remains inaccessible.

In all the following, two angular quantities are considered:

- $\cos \theta^{\star}$ is the angle between the outgoing electron and the quark, in the $Z^{\prime}$ rest frame, deduced from the generator history.

- $\cos \theta^{\otimes}$ is the angle between the outgoing electron candidate, in the reconstructed $Z^{\prime}$ rest frame, and the direction of the reconstructed $Z^{\prime}$.

The first quantity will be used to extract the reference asymmetry, whereas the second one will be used as an estimator; the correlation between the two quantities is represented on Fig. 1, two main populations of events being exhibited:

- when the quark is in the same direction as the $Z^{\prime}$, the $\cos \theta^{\otimes}$ quantity is an unbiased estimator of the $\cos \theta^{\star}$ angle, degraded only by initial state radiation, energy and angular resolution. As the $Z^{\prime}$ is produced by the annihilation of a quark and a sea antiquark, this configuration is the most frequent.

- if not, the estimator is strongly biased, with $\cos \theta^{\otimes} \approx-\cos \theta^{\star}$

The relative proportion $\varepsilon$ of the second population of events can be estimated as a function of the rapidity and is represented on figure 1 . 

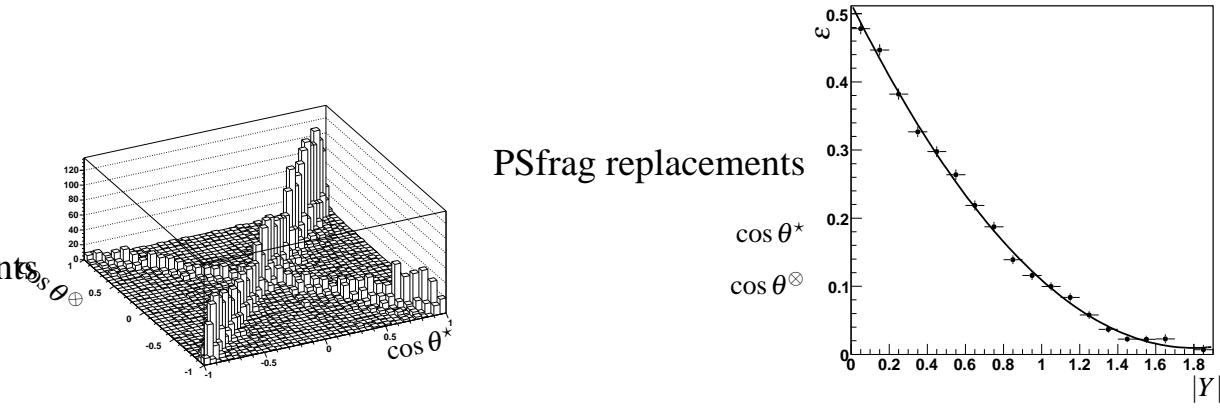

Figure 1: Left: $\cos \theta^{\star}$ vs $\cos \theta^{\otimes}$; Right: $\varepsilon(|Y|)$ at $M=1.5 \mathrm{TeV}$ (with a parabolic parametrisation superimposed)

\section{The $A_{F B}$ extraction}

Given relation 1.1, the $A_{F B}$ parameter can be classicly extracted at the generation level by a counting method:

$$
A_{F B}^{g e n}=\frac{N_{+}-N_{-}}{N_{+}+N_{-}}
$$

where $N_{+}\left(N_{-}\right)$denotes the number of events with $\cos \theta^{\star}>0(<0)$.

Applying the same formula to measure the observed asymmetry by taking $\cos \theta^{\otimes}$ as an estimator of $\cos \theta^{\star}$ leads to a bias due to the imperfect knowledge of the quark direction. Ignoring the detector effects and the $Z^{\prime}$ transverse momentum, this bias can be quantified with the knowledge of the $\varepsilon$ proportion defined in section 1 :

$$
A_{F B}^{o b s}=(1-2<\varepsilon>) A_{F B}^{g e n}
$$

A corrected asymmetry is then defined by:

$$
A_{F B}^{c o r}=\frac{1}{1-2<\varepsilon>} A_{F B}^{o b s}
$$

This method is very easy to implement and efficient but the whole angular information is unfortunately lost, when integrating over an hemisphere; the spin 1 behaviour of the new particle is consequently not tested at all. To allow such a test, a differential analysis is performed : a differential asymmetry is deduced by a counting method, the quantity $N_{ \pm}$being extracted on a limited $\cos \theta^{\star}\left(\right.$ or $\left.\cos \theta^{\otimes}\right)$ range. The running of this quantity can be easily deduced from expressions (1.1) and (2.1):

$$
A_{F B}(\cos \theta)=\frac{8}{3} A_{F B} \times \frac{\cos \theta}{1+\cos ^{2} \theta}
$$

Performing an analytical $\chi^{2}$ fit to these differential asymmetry distributions in the $[0,1]$ interval then allows to extract the $A_{F B}^{g e n}, A_{F B}^{o b s}$, and $A_{F B}^{c o r}$ quantities.

\section{Application to the ATLAS case}

In order to validate the above method, Monte Carlo event samples have been considered; they have been produced by the PYTHIA generator[4], the ATLAS detector response being simulated 
in the Geant3 framework. Several models involving a new gauge boson of mass $1.5 \mathrm{TeV}$ have been considered : $\chi, \psi, \eta$ (all derived from a $E_{6}$ theory) and a left right symmetric model. Given the ATLAS detector performances, the signal signature should be very clean (two highly energetic leptons) and the background should remain limited; no background was therefore included in this study.

The three asymmetries detailed in the section 2 are represented on figure 3 for the $\chi$ model in 5 different dileptons mass bins, and for a luminosity of $100 \mathrm{fb}^{-1}$. The 5 - independent- measurements exhibit the same behaviour : due to the imperfect knowledge of the incoming quark direction, the observed asymmetry is much lower in absolute value than the one computed at the generation level. Applying the adequate $\varepsilon$ correction factor however allows to recover from this effect. Due to the different coupling constants to quarks, a systematic error must be associated to the estimate of the $\varepsilon$ quantity; the impact on the $A_{F B}$ measurement was found to be limited, below $10 \%$.

Finally, applying this method on all available samples leads to identical conclusions; on figure 3 , are represented all the corrected asymmetries; the variety of behaviours is a clear proof of the discrimination potential of this observable.
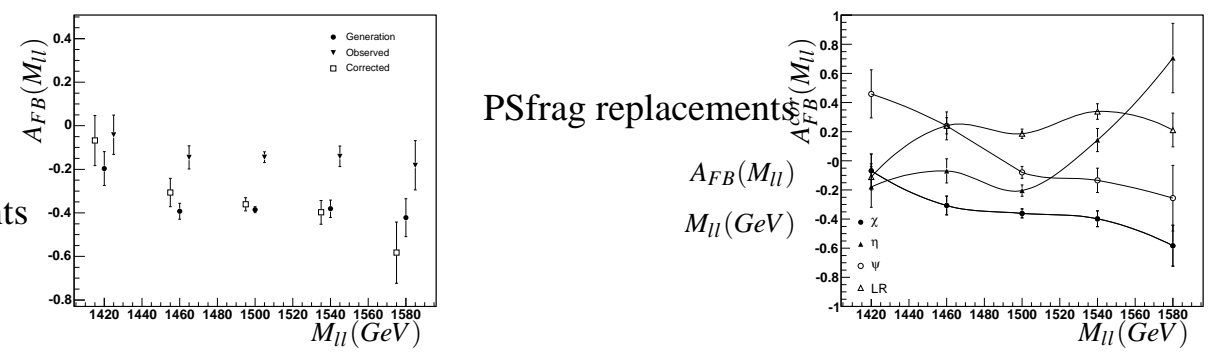

Figure 2: Left: Asymmetries measurement $-\chi$ model $\left(M_{Z}^{\prime}=1.5 \mathrm{TeV}\right)-\int \mathscr{L}=100 \mathrm{fb}^{-1}$; Right: Corrected asymmetry $-\int \mathscr{L}=100 \mathrm{fb}^{-1}$

\section{Conclusion}

A method to measure the leptonic forward-backward asymmetry at the LHC was presented. The accuracy reached with a realistic luminosity was found to be promising; this measurement, associated with the one of other observables (natural width, rapidity) should provide large constraints on the model at the origin of any -potential- new massive gauge boson.

\section{References}

[1] The CDF collaboration, Search for new particles decaying to high mass dielectrons or dimuons at CDF II, CDF note 7286

[2] The D0 collaboration, Search for heavy $Z^{\prime}$ boson in the dielectron channel with $200 \mathrm{pb}^{-1}$ of data with the D0 detector, D0 note 4375

[3] The ATLAS collaboration, ATLAS Detector and physics performance technical design report, volume II, CERN/LHCC/99-15 (1999)

[4] T.Sjöstrand et al., PYTHIA 6.2 Physics and Manual, Comp. Phys. Comm. 135 (2001) 238 [hep-ph/0108264] 